\title{
A review about oral health and dental care among the aged population
}

\author{
Elaheh Aghaei', Mohammad Vahedi ${ }^{2}$, Arezoo Alaee $D * 3$ \\ ${ }^{1}$ Member of Oral Medicine Dept,Dental Material Research Center,Faculty of Dentisry, Tehran Medical \\ Science Islamic Azad University, Tehran, Iran. \\ ${ }^{2}$ Oral Medicine Dept, Faculty of Dentisry, Tehran Medical Science Islamic Azad University, Tehran, Iran. \\ ${ }^{3}$ Oral Medicine Dept, Member of Dental Material research Center, Faculty of Dentisry, Tehran Medical \\ Science Islamic Azad University, Tehran, Iran.
}

\section{ARTICLE INFO}

\section{Article History}

Received:March 2021

Accepted: Apr 2021

ePublished: June 2021

Corresponding author:

Arezoo Alaee,Member of Oral Medicine Dept,Dental Material Research Center,Faculty of Dentisry, Tehran Medical Science Islamic Azad University, Tehran, Iran.

Email: arezoo.alaee@ yahoo.com

\section{ABSTRACT}

Background and Aim: The population of the elderly is growing, and oral health of this specific population is suboptimal because the prevalence of caries, xerostomia, edentulism, and oral cancer has increased among them. Impaired oral health may cause many problems and negatively affect the quality of life (QoL). Due to the increased need for preventive and restorative dental procedures with aging, effective strategies must be implemented in this respect. Thus, it is necessary to assess the oral health status and dental care needs of the geriatric population.

Materials and Methods: A literature search was conducted for studies published between 2015 and 2020 in the PubMed, Nature, Wiley, Science Direct and Google Scholar. Of 72 articles identified, 26 articles that were case reports and case series or had irrelevant titles or keywords were excluded, and 46 articles remained for data extraction.

Results: The most common oral problems in the elderly included xerostomia, oral pain, impaired deglutition, and mastication problems. Also, they suffered from edentulism, orofacial pains, pneumonia, and cognitive problems. Thus, they may need special dental care.

Conclusion: Provision of dental care must be promoted to ensure that older people have appropriate access to oral health services. Many items affect the oral healthrelated quality of life (OHRQoL) of the elderly. Thus, dentists, dental hygienists, caregivers, and geriatricians must focus on improving the elderly's oral health status. Oral health promotion programs in the elderly can decrease their oral problems and improve their OHRQoL.

J Res Dent Maxillofac Sci 2021;6(3):30-38.

\section{Keywords: Oral Health; Aged; Dental Care; Preventive Health Services}

\section{Introduction}

Many societies, particularly industrialized countries, are aging due to the decreasing birth rate and increased life expectancy. Based on the data of the Statistical Center of Iran, about $9.3 \%$ of the population were over 60 years in 2015. ${ }^{(1)}$

The World Health Organization estimates that 20$25 \%$ of the population of Iran will be elderly by the year $2050 .^{(2)}$ Such a significant demographic transition in population will have great impacts on the health care system. As people get older, staying healthy becomes more challenging since the elderly increasingly become care-dependent and frail.
Older people, especially those who are caredependent and frail, often have poor oral health and poor oral hygiene.

As a population ages, the incidence of chronic illnesses and limitations increases. Older adults are at an increased risk for almost all chronic illnesses which may lead to both morbidity and mortality. Thus, it is important to find how the elderly adapt themselves to age-related changes. ${ }^{(3)}$

Oral health is a fundamental part of general health. The elderly may need special preventive and curative oral care. 
The most common oral diseases among the aged people include dental caries, periodontitis, dry mouth, tooth loss, and oral cancer. Thus, the need for preventive and restorative dental procedures increases with aging ${ }^{(4)}$, and effective preventive and therapeutic procedures must be provided to the elderly. ${ }^{(3)}$ The present study aims to review the available data about the oral health status and dental care needs of the elderly and the preventive dental services provided to them.

\section{Materials and Methods}

A literature search was conducted in the PubMed, Nature, Wiley, Science Direct and Google Scholar, which was limited to English articles published between 2015 and 2020. The following search terms were used: Aged, frail elderly, older adults, elderly patient, gerodontology, older people, functionally dependent elderly, dental care, residential aged care, oral mucosal lesion, and soft tissue lesions. Of 72 articles identified in the search, 26 articles that were case reports and case series, or had irrelevant titles or keywords were excluded, and 46 articles remained for data extraction.

\section{Results}

The most common oral changes in the elderly included xerostomia, oral pain, swallowing, and mastication problems. Also, they had salivary gland hypofunction, edentulism, orofacial pain, pneumonia due to aspiration, and cognitive problems. Thus, they may need special dental care.

\section{Discussion}

Geriatric dentistry:

Geriatric dentistry includes all features of oral health and dental care of the geriatric population. Oral health refers to acceptable oral function and absence of disease $\mathrm{e}^{(4)}$ The population is aging, and older adults are retaining their natural teeth more than before, which leads to an increased risk of dental caries and periodontal disease. ${ }^{(5)}$ Also, the older population may need specific preventive and therapeutic care. ${ }^{(4)}$ Oral disorders may also aggravate with aging. Oral diseases in the elderly can lead to pain and reduced masticatory function, and negatively affect their physical appear- ance and speech. In addition, they could affect general health and increase the risk of endocrine, neurological, respiratory, and cardiovascular diseases. Oral health problems may cause changes in diet, decrease nutritional quality, and decrease the quality of life (QoL). ${ }^{(6)}$ Older people, particularly care-dependents, may have problems accessing dental care. We need to understand the oral health requirements of the residents of nursing homes and develop strategies to manage their problems to ensure that this group can access dental services easily. ${ }^{(5)}$

\section{Oral health-related quality of life:}

It has been confirmed that in the geriatric population, oral health condition is related to oral health-related quality of life (OHRQoL). To improve the OHRQoL of the elderly, it is important to improve their daily oral care, reduce the rate of caries, and treat their oral conditions. As good oral health is necessary for healthy aging, it is essential to organize a teamwork of different specialists for this purpose. ${ }^{(6)}$ The factors related to OHRQoL of the elderly include smoking, educational level, number of functional occluding teeth, drinking and brushing habits, etc. ${ }^{(7)} \mathrm{A}$ cross-sectional study found that oral health had an impact on the QOL of the institutionalized and non-institutionalized aged people. Lower OHRQoL of institutionalized older people is associated with lower physical and psychological status. ${ }^{(8)}$ Factors related to poor OHRQoL include self-reported oral health problems, edentulism, frailty, depression, retiring ${ }^{(8)}$, and female gender. ${ }^{(6)}$ Generally, perceived oral health is better in home-dwellers, and they are able to bite and chew more easily. ${ }^{(9)}$ Due to the association between the self-perceived oral health and OHRQoL in aged people, it is important to detect oral health problems as soon as possible specifically swallowing disorders among the frail older people. ${ }^{(6)}$ A cross-sectional study showed that oral health data are related to self-perception of general and oral health. ${ }^{(10)}$ According to a recent study, clinical evaluations of oral health differed remarkably from the satisfaction of older people with their oral health. In this study, a large majority of the older people expressed that they were pleased with their oral health condition, while only a minority of them were identified without oral prob- 
lems. This inconsistency indicates that to provide more person-centered care, both professional assessment and asking the elderly about their selfperceived oral health are equally important. ${ }^{(11)}$ Factors such as mastication, brushing teeth after every meal and before sleep, and use of dentures impact on self-rated oral health. Self-rated health is also a psychological factor. General and socioeconomic features such as age, gender, educational level, living status, monthly income, oral health behavior, oral function, and OHRQoL are the independent variables that affect oral health. Self-rated oral health is higher in males than in females. ${ }^{(12)}$ Self-reported oral health is definitely affected by general health and socioeconomic status. People with a higher socioeconomic status and higher educational level usually pay more attention to their oral health, resulting in fewer oral problems. ${ }^{(11,12)}$ The number of people retaining their natural teeth to an old age is rapidly growing, and this predictable increase in number will have a considerable effect on the oral health requirements of the older people. ${ }^{(13)}$ Based on the result of a Canadian cross-sectional study, poor oral health is associated with smoking, low income, low social support, low physical training, and lack of dental visits. ${ }^{(14)}$

Care-dependent elderly retaining their teeth are mainly less frail, have a better physical function, and better general health and QoL than edentulous aged people. One study compared the oral health status of the elderly retaining their natural teeth and edentulous elderly and their cooperation for dental treatments and showed that in comparison with the edentulous elderly, the other group appeared younger at admittance, had greater need for oral health care and poorer oral hygiene, and were less cooperative for oral care. ${ }^{(13)}$ Most older people who live in nursing homes are not able to clean their teeth or denture correctly and also due to transportation problems, they cannot visit a dentist, which results in poor oral health. ${ }^{(13)} \mathrm{A}$ cross-sectional study explained that coexistence of tooth loss and fast eating increased the risk of metabolic syndrome in the aged population. In contrast, oral health instruction and retaining the teeth prevented the metabolic syndrome in the geriatric population. In addition, daily usage of oral hygiene products reduced the risk of metabolic syndrome ${ }^{(15)}$
Oral and perioral tissues undergo distinct functional and structural changes as a person ages. The frequency of oral lesions in the elderly is high ${ }^{(16)}$, and is associated with the usage, quality, and hygiene level of the mandibular and maxillary removable dentures. Utilization of an ultrasonic bath is an effective and simple way to improve denture hygiene in the elderly who have dementia or need special oral care. ${ }^{(17)}$ It is necessary to have diagnostic and preventive measures and educate the older people for early screening of any oral mucosal lesions particularly premalignant and malignant lesions.

\section{Oral health assessments:}

Good oral health is necessary for the wellbeing of the aged population. Most older residents in nursing homes have poor to medium oral health conditions. An accurate assessment of oral health should be included in general health assessments of the geriatric population. To design a better health care plan for the aged population, dentists and specialists should use the oral health and general health records of the patients. ${ }^{(18)}$

Several instruments have been developed in the past 20 years to measure the OHRQoL. ${ }^{(19)}$ The most commonly used questionnaires for measuring the impact of oral health on the QOL are the Oral Health Impact Profile and its derivatives and the Geriatric Oral Health Assessment Index (GOHAI). The GOHAI was first designed by the American scholars in 1990. ${ }^{(20)}$ This valid, reliable, and useful questionnaire is a self-reported measure frequently used to assess the OHRQoL in the care-dependent and care-independent elderly. ${ }^{(21)}$ It consists of the following items: speech, eating and swallowing, self-perceived oral health, concerns about oral health, avoidance of social contact due to oral problems, dissatisfaction with

the appearance, and use of pain killers for oral pain. ${ }^{(7)}$ Using self-rated questions is a simple way to help professionals acquire better knowledge about the health status of their patients and the important aspects that can impact on their lives. ${ }^{(10)} \mathrm{A}$ recent study about self-perception of oral health among the aged people indicated that GOHAI is associated with systemic conditions such as the number of comorbid conditions, the number of medications taken, and BMI. 
BMI has an inverse correlation with the GOHAI score and the lower the BMI, the higher the GOHAI score would be. But there is no correlation between the observed oral health status and GOHAI score, such as the number of teeth, dry mouth, or denture status. ${ }^{(22)}$ he self-rated oral health of the older people is based on psychological factors, demographic and sociological aspects, oral health behavior, oral function, and physical function. ${ }^{(12)}$

Other instruments to measure the OHRQoL include the Oral Health Assessment Tool ${ }^{(23)}$ and Oral Health Impact Profile. ${ }^{24)}$

\section{Oral health and general health:}

Physical and mental disorders, chronic illnesses, and other comorbidities are common in the geriatric population. Systemic diseases impact on oral status. Also, poor oral health status negatively affects the general health. ${ }^{(9)} \mathrm{A}$ cross-sectional and longitudinal study showed that older people who have a poorer oral health status have poorer general health as well. About $80 \%$ of the general health indicators can be predicted from oral health indicators. Having carious teeth is a predictor of poor activities of daily living in the future. ${ }^{(18)}$ Oral status is associated with general health, frailty, and QoL. The oral health of the older people declines during sickness. Therefore, healthcare workers must prevent poor oral health status causing general health problems in the residents of nursing homes. The elderly that retain teeth mainly perform better; thus, maintaining a functional dentition and good oral health decrease the likelihood of general health problems. ${ }^{(25)}$

\section{Oral health issues}

Dry mouth, oral pain, swallowing and chewing problems, and a feeling of insecurity are the most common oral health issues among the elderly. ${ }^{(26)}$ Ahmad et al. reported that age had an inverse correlation with the saliva flow and $\mathrm{pH}$. The $\mathrm{pH}$ and flow rate of the saliva decrease with aging. Both low salivary $\mathrm{pH}$ and hyposalivation contribute to a poorer QoL. Besides, old people who have low salivary flow rate, will have higher caries prevalence and it has been reported that high caries prevalence is associated with poorer QoL. ${ }^{(27)}$

Aging is accompanied by numerous clinical problems, including a pro-inflammatory state, that is directly related to the microbiota of older adults enhancing the susceptibility to disease. Veillonella atypica, Prevotella histicola, Streptococcus parasanguinis, and Streptococcus salivarius are more dominant in the elderly with fewer teeth, more dental caries experience, and higher plaque index. These results suggest that the elderly with poorer oral health swallow a more dysbiotic microbiota formed on the tongue. Aspiration of oral contents can lead to pneumonia, increasing the risk of death in the elderly. Pneumonia is a major cause of death among the elderly. if cleaning of dentures is performed daily, it may decrease the risk of pneumonia. ${ }^{(28)}$ The results of a cohort study in Korea showed that improving oral health may diminish the risk of pneumonia in these people.(29)

Dependent persons have a poor oral health, which has a negative impact on their masticatory function and OHRQoL. ${ }^{(30)}$ Older adults who require help for self-care have much higher risk of having oral problems and higher frequency of food debris and broken teeth. ${ }^{(11)}$ Xerostomia is a common oral problem in older people, which has been attributed to chronic diseases and the use of medications. ${ }^{(28)}$ Smoking is another factor causing oral health problems. Smoker elderly are more susceptible to dental caries compared with non-tobacco users. ${ }^{(28)}$

Aging is associated with a change in chewing ability, ${ }^{(18)}$ decreased tongue pressure, and impaired motor function of the lips. ${ }^{(31)} \mathrm{A}$ prospective cohort study reported that poor oral health and deglutition dysfunction are independent mortality risk factors of the elderly in intermediate care. Therefore, systematic monitoring of the swallowing function and oral health status of the elderly in intermediate care can help improve care planning. ${ }^{(32)}$ Since the elderly have difficulties in mastication and swallowing, prolonged meal consumption leads to a decline in the swallowing function. ${ }^{(31)}$ Older people with swallowing problems are more likely to have poor OHRQoL. ${ }^{(6)}$

Malnutrition in the elderly is associated with oral health complaints such as chewing problems ${ }^{(33)}$ while the results of a cross-sectional 
study showed that malnutrition among the aged people was not related to their oral health status. (34) Poor oral health is related to poorer diet quality, which may lead to higher frailty. ${ }^{(14)}$

There is a significant correlation between the oral health status and preserved cognitive functions. ${ }^{(34)}$ Orofacial pain is frequently present in individuals with mild cognitive impairment or dementia. Numerous factors cause poor oral health in geriatric patients su $\square$ ering from dementia such as behavioral disorders, cognitive function deterioration, and insufficient medical sta-training regarding oral hygiene. It has been demonstrated that the lower the degree of dementia, the lower tends to be the oral health status. Development of dementia can be related to tooth loss. There is an association between dental caries severity and cognitive disabilities among the cognitively disabled patients. ${ }^{(35)}$ Alzheimer patients have a poor oral health and high rate of caries and periodontal disease, poor saliva quantity and quality, and more mucosal lesions including candidiasis and cheilitis. ${ }^{(36)}$ Frailty is defined as a condition in which older adults are vulnerable to sudden changes in health status because of a decline in physiological function and reserve. ${ }^{(37)}$ Many old people with systemic conditions often need support from caregivers to visit a dentist. ${ }^{(6)}$ The greater the frailty, the more difficult it would be to visit a dentist, resulting in poorer oral health ${ }^{(26)}$

Frailty is associated with a lower number of remaining teeth while using dentures cannot prevent frailty in the elderly with fewer teeth. Poor oral health and oral pain have a harmful effect on the QoL, and can limit social interactions. (38) Some elderly people do not visit a dentist because they have other health problems that require more attention. ${ }^{(26)}$ According to a recent population-based cohort study, the more the oral health problems, the greater the risks of being frail and developing frailty in older age would be. Dry mouth, tooth loss, and other oral health problems could be significant predictors of frailty in older people. The identification and management of poor oral health in older people could be important in preventing frailty. ${ }^{(39)}$ Poor oral health can affect oral intake and nutritional status; thus, it could also serve as an important risk factor for frailty. ${ }^{(39)}$ Risk of oral problems is high in the elderly with some remaining teeth and those with dental implants to support their overdenture, especially when oral hygiene maintenance and dental visits become difficult due to frailty. But even complete dentures can become problematic when the denture fit is poor. There is no standard way to prevent frailty. A combination of oral health instruction, physical exercise, psychosocial health care, and a dietary approach may be appropriate to improve the health status of the older people. ${ }^{(40)}$

\section{Access to dental care}

Oral health problems in nursing homes are a consequence of barriers against oral healthcare, challenges in managing the oral health of residents, and poor skills of the facility staff. The difficulty with the provision of dental care to older people is one of the biggest issues faced by the dental health workers. ${ }^{(5)}$ Due to the enhancement of oral health over the last 60 years, the population of adults aged 65 years and older is growing exponentially and because of the decreasing rate of edentulism and consequently an increasing number of teeth present, this population have rapidly changing dental needs. This is a challenge for the public dental services, dental care providers, public health policymakers, and dental education providers ${ }^{(5)}$ to develop models of care In order to improve access to dental care for the functionally dependent and frail elderly. Thus, it is essential to increase awareness on the benefits of having a healthy oral cavity. Knowledge enhancement must be associated with regular clinical examinations. ${ }^{(9)}$ According to a recent study in Sweden, the healthcare providers did not have the essential knowledge of oral health mandatory for their responsibility. Besides, they implied that their own attitudes were important when attending to the oral health of the elderly. This highlights the importance of participation of all healthcare providers in an educational program, to effectively enhance their dental care knowledge. ${ }^{(41)}$

\section{Oral hygiene:}

Oral health behaviors reflect general lifestyle habits. ${ }^{(15)}$ The elderly often abandon oral health because of resignation, loss of motor and cognitive abilities, or sensory loss through a decline in their five senses. ${ }^{(9)}$ Older people who brush their teeth frequently, often have a healthier lifestyle. Tooth brushing and the use of secondary oral hygiene products prevent oral diseases. ${ }^{(15)}$ Accord- 
ing to a recent study, the higher the frequency of brushing, the higher the score for happiness. ${ }^{(12)}$ The sanitary system in the nursing homes is insufficient, or the oral health care team does not have adequate knowledge about oral hygiene maintenance. ${ }^{(28)}$ Monthly professional oral care, associated with individual oral health care instructions, may reduce root caries and improve oral hygiene among the nursing home residents. This may also lead to a more positive attitude towards oral hygiene, in comparison with common daily oral care. ${ }^{(42)}$ Professional cleaning has a positive impact on gingival bleeding. Besides, Individual oral hygiene instruction leads to dental plaque reduction; therefore, both individual instruction and education must be included in oral health care programs. ${ }^{(43)}$ Lack of daily tooth brushing leads to an increase in the DMFT score in older people and those practicing tooth brushing have lower oral health index. Because of the low rate of daily brushing, a preventive plan to alleviate the oral diseases in older people is necessary. ${ }^{(28)}$ Gingival health and oral hygiene may be worsened with the decrease in cognition and dental-related functions. Dental plaque scores are higher in more dependent patients. ${ }^{(36)}$

\section{Oral health care in nursing homes}

The older people who are residents of a nursing home have a poor oral health condition and high needs for dental and prosthetic treatments. ${ }^{(4)}$ A Massachusetts survey disclosed that $34 \%$ of geriatric patients in nursing homes had critical and important dental needs. ${ }^{(5)}$ Furthermore, they may have more difficulties accessing oral health services and also suffer more from poor oral health status in comparison with the aged people living in their homes. ${ }^{(34)}$ A recent study reported that the prevalence of polypharmacy increased with age, leading to complicated care needs in the elderly. ${ }^{(45)}$ Individuals 90 years of age have great preventive and therapeutic dental care needs that affect their QoL while they are seldom aware and lack transportation. These people need to get assistance with oral hygiene and regular visits with a dentist in order to prevent oral problems in them and improve their adaptation to removable prostheses or alternative rehabilitation treatments. ${ }^{(4)}$ Regular clinical oral examination of old people would improve their oral health. In nursing homes, physicians are responsible for visiting the patients regularly. Researchers observed that the use of oral care in old patients decreases, particularly in institutions. ${ }^{(9)}$ Knowledge on oral health status of geriatric patients is lacking. It has not been confirmed that the oral health status of the elderly living in nursing homes differs from that of other older people. ${ }^{(25)}$ To improve the oral health of this specific population, we need to provide personcentered oral care. ${ }^{(11)}$ Financial problems are the main cause of forgoing of medical care specially dental care among the aged population. ${ }^{(9)}$

Rosa et al. ${ }^{(12)}$ suggested that to improve the oral health of the geriatric population, we should enhance home-based interventions or tele-dentistry to increase the accessibility of dental services and promote preventive and minimally invasive treatments. In addition, dental caregivers must be aware of the value of oral health.

Generally, the oral health condition of the geriatric patients who live in nursing homes is an important issue for the healthcare system because maintaining good oral health is a critical component of general health. ${ }^{(34)}$

\section{Preventive treatment planning}

Considering the increasing number of aged individuals, the need for preventive dental care increases. Alaee et al. stated that the knowledge of dentists about geriatric dentistry is insufficient and due to their positive attitude towards geriatric dentistry, it is necessary to design and implement suitable educational courses for dental students and organize conferences and continuing education courses for dentists about geriatric dentistry. $^{(46)}$ Also, health education plans should be developed for the elderly. ${ }^{(12)}$

Oral rehabilitation can promote the health status and QoL of the elderly and decrease the risk of oral problems in them. To improve oral rehabilitation outcomes, prevention and early identi

fication of oral problems and advanced medical and dental care are necessary. ${ }^{(47)}$

The use of oral health therapists in aged care facilities can improve the oral health of nursing home residents including their plaque index. (47) It has been observed that the application of an integrated oral health program including oral health therapists and tele-dentistry could improve the gral health status of nursing home ${ }_{35}$ 
residents and provide better access to oral health education for them. Also, this efficient model can help the elderly with dementia and other high care conditions in order to enhance their access to oral health services. Tele-dentistry is suitable for older people who have complications that would impact on dental visits such as inability to go for a dental visit. ${ }^{(48)}$ Utilization of a mobile dental team leads to a considerable increase in knowledge and attitude of the care givers regarding oral health. Developing an oral health professional team in nursing homes could integrate oral health into general care and remove barriers against daily oral hygiene. ${ }^{(49)}$ Iwao et al. stated that participation in long-term preventive programs combined with physical exercise, nutritional guidance, and oral health instruction may contribute to enhanced oral and physical function such as improvement of swallowing and decreased risk of dysphagia in the elderly, especially at a younger age. As this program decreases the oral bacterial count, it can improve the oral hygiene status. ${ }^{(50)}$ Kossioni et al. ${ }^{(3)}$ explained the main areas for more action by policymakers including oral health educational plans for the aged people, health plans including prevention, and easy access to dental care, assessment of both oral health and general health, oral health promotion in organized settings, and citizen authorization for involvement in oral care actions of the older people.

\section{Conclusion}

The oral health status of the geriatric population is generally poor. The prevalence of caries and tooth loss has increased which leads to mastication difficulties and impairment of OHRQoL, with direct effects on general QoL and well-being. Dental practice must be promoted to ensure that older people have appropriate access to oral health services. Many items affect the OHRQoL; thus, oral health workers including dentists, dental hygienists, caregivers, and geriatricians must concentrate on improving the elderly's oral health status. Oral health promotion programs in the elderly can cause a reduction in oral problems and improve OHRQoL.

\section{Acknowledgement:}

The authors declare no competing interests.

\section{References}

1. Statistical Center of Iran. 2015.

2. World Health Organization, Regional office for the eastern Mediterranean. 2011.

3. Kossioni AE, Hajto-Bryk J, Maggi S, McKenna G, Petrovic M, Roller-Wirnsberger RE, Schimmel M, Tamulaitienè M, Vanobbergen J, Müller F. An Expert Opinion from the European College of Gerodontology and the European Geriatric Medicine Society: European Policy Recommendations on Oral Health in Older Adults. J Am Geriatr Soc. 2018 Mar;66(3):609-613.

4. Rosa RW, Samot J, Helmer C, Pourtau G, Dupuis V, Fricain JC, Georget A, Dartigues JF, Arrivé E. Important oral care needs of older French people: A cross-sectional study. Rev Epidemiol Sante Publique. 2020 Apr;68(2):8390.

5. Hopcraft MS. Dental demographics and metrics of oral diseases in the ageing Australian population. Aust Dent J. 2015 Mar;60 Suppl 1:2-13.

6. Koistinen S, Olai L, Ståhlnacke K, Fält A, Ehrenberg A. Oral health-related quality of life and associated factors among older people in short-term care. Int J Dent Hyg. 2020 May;18(2):163-172.

7. Shao R, Hu T, Zhong YS, Li X, Gao YB, Wang YF, Yin W. Socio-demographic factors, dental status and health-related behaviors associated with geriatric oral health-related quality of life in Southwestern China. Health Qual Life Outcomes. 2018 May 21;16(1):98.

8. Farias IPS, Montenegro LdAS, Wanderley RL, de Pontes JCX, Pereira AC, de Almeida LdFD, et al. Physical and psychological states interfere with health-related quality of life of institutionalized elderly: a cross-sectional study. BMC geriatrics. 2020;20(1):1-10.

9. Maille G, Saliba-Serre B, Ferrandez A-M, Ruquet M. Use of care and the oral health status of people aged 60 years and older in France: results from the National Health and Disability Survey. Clinical interventions in aging. 2017;12:1159-66.

10. TÔrres LHDN, Fagundes MLB, Silva DDD, Neri AL, Hilgert JB, Hugo FN, Sousa MDLR. Self-rated general and oral health and associated factors in independently-living older individuals. Braz Oral Res. 2020;34:e079.

11. Koistinen S, Olai L, Ståhlnacke K, Fält A, Ehrenberg A. Oral health and oral care in short-term care: prevalence, related factors and coherence between older peoples' and professionals' assessments. Scand J Caring Sci. 2019 Sep;33(3):712-22.

12. Moon J-H, Heo S-J, Jung J-H. Factors Influencing SelfRated Oral Health in Elderly People Residing in the Community: Results from the Korea Community Health Survey, 2016. Osong Public Health and Research Perspectives. 2020;11(4):245-50.

13. Hoeksema AR, Peters LL, Raghoebar GM, Meijer HJA, Vissink A, Visser A. Oral health status and need for oral care of care-dependent indwelling elderly: from admission to death. Clin Oral Investig. 2017 Sep;21(7):2189-96.

14. Bassim C, Mayhew AJ, Ma J, Kanters D, Verschoor CP, Griffith LE, Raina P. Oral Health, Diet, and Frailty at Baseline of the Canadian Longitudinal Study on Aging. J Am Geriatr Soc. 2020 May;68(5):959-66.

15. Saito M, Shimazaki Y, Nonoyama T, Tadokoro Y. Num- 
ber of teeth, oral self-care, eating speed, and metabolic syndrome in an aged Japanese population. Journal of epidemiology. 2019;29(1):26-32.

16. Cheruvathoor DD, Thomas V, Kumar NR, Jose M. High prevalence of oral mucosal lesions in elderly: Call for revolutionizing geriatric dental care strategies. J Family Med Prim Care. 2020 Aug 25;9(8):4375-80.

17. Zenthöfer A, Meyer-Kühling I, Hufeland A-L, Schröder J, Cabrera T, Baumgart D, et al. Carers' education improves oral health of older people suffering from dementia-results of an intervention study. Clinical interventions in aging. 2016;11:1755-62.

18. Tran TD, Krausch-Hofmann S, Duyck J, de Almeida Mello J, De Lepeleire J, Declerck D, et al. Association between oral health and general health indicators in older adults. Scientific reports. 2018;8(1):1-6.

19. Sischo L, Broder HL. Oral health-related quality of life: what, why, how, and future implications. J Dent Res. 2011 Nov;90(11):1264-70.

20. Atchison KA, Dolan TA. Development of the Geriatric Oral Health Assessment Index. J Dent Educ. 1990 Nov;54(11):680-7.

21. Aguirre-Bustamante J, Barón-López FJ, CarmonaGonzález FJ, Pérez-Farinós N, Wärnberg J. Validation of a modified version of the Spanish Geriatric Oral Health Assessment Index (GOHAI-SP) for adults and elder people. BMC Oral Health. 2020 Feb 19;20(1):61.

22. Mendes MSS, Chester LN, Fernandes Dos Santos JF, Chen X, Caplan DJ, Marchini L. Self-perceived oral health among institutionalized older adults in Taubate, Brazil. Spec Care Dentist. 2020 Jan;40(1):49-54.

23. Klotz AL, Zajac M, Ehret J, Hassel AJ, Rammelsberg P, Zenthöfer A. Development of a German version of the Oral Health Assessment Tool. Aging Clin Exp Res. 2020 Jan;32(1):165-72.

24. Kireilytė M, Masiliūnaitė V, Belickienė V, Žilinskas J, Sakalauskienė Ž. Testing of Lithuanian version of the Oral Health Impact Profile-14 among older adults. A Pilot study. Stomatologija. 2019;21(3):67-71.

25. Hoeksema AR, Peters LL, Raghoebar GM, Meijer HJA, Vissink A, Visser A. Health and quality of life differ between community living older people with and without remaining teeth who recently received formal home care: a cross sectional study. Clin Oral Investig. 2018 Sep;22(7):2615-22.

26. Bakker MH, Vissink A, Spoorenberg SLW, Wynia K, Visser A. Self-reported oral health problems and the ability to organize dental care of community-dwelling elderly aged $\geq 75$ years. BMC Oral Health. 2020 Jul 2;20(1):185.

27. Ahmad MS, Bhayat A, Zafar MS, Al-Samadani KH. The impact of hyposalivation on quality of life (QoL) and oral health in the aging population of Al Madinah Al Munawarrah. International journal of environmental research and public health. 2017 Apr;14(4):445.

28. Choufani A, Folliguet M, El-Osta N, Rammal S, Doumit M. Oral health status and care of institutionalized elderly individuals in Lebanon. Indian J Dent Res. 2020 JulAug;31(4):507-14.

29. Son M, Jo S, Lee JS, Lee DH. Association between oral health and incidence of pneumonia: a population-based cohort study from Korea. Sci Rep. 2020 Jun; 10: 9576.

30. Baumgartner W, Schimmel M, Müller F. Oral health and dental care of elderly adults dependent on care. Swiss Dent J. 2015;125(4):417-26.

31. Hiramatsu T, Kataoka H, Osaki M, Hagino H. Effect of aging on oral and swallowing function after meal consumption. Clin Interv Aging. 2015 Jun;10:229-35.

32. Hägglund P, Koistinen S, Olai L, Ståhlnacke K, Wester P, Levring Jäghagen E. Older people with swallowing dysfunction and poor oral health are at greater risk of early death. Community Dent Oral Epidemiol. 2019 Dec;47(6):494-501. 33. Bakker MH, Vissink A, Spoorenberg SL, Jager-Wittenaar H, Wynia K, Visser A. Are Edentulousness, Oral Health Problems and Poor Health-Related Quality of Life Associated with Malnutrition in Community-Dwelling Elderly (Aged 75 Years and Over)? A Cross-Sectional Study. Nutrients. 2018 Dec; 10(12): 1965.

34. Chiesi F, Grazzini M, Innocenti M, Giammarco B, Simoncini E, Garamella G, Zanobini P, Perra C, Baggiani L, Lorini C, Bonaccorsi G. Older People Living in Nursing Homes: An Oral Health Screening Survey in Florence, Italy. Int J Environ Res Public Health. 2019 Sep 19;16(18):3492.

35. Chen X, Clark JJ, Chen H, Naorungroj S. Cognitive impairment, oral self-care function and dental caries severity in community-dwelling older adults. Gerodontology. 2015 Mar;32(1):53-61.

36. Chen X, D'Souza V, Yu L. The oral health status of residents with different cognitive and dental-related functions in three North Carolina assisted living facilities. Gerodontology. 2019 Jun;36(2):142-8.

37. Clegg A, Young J, Iliffe S, Rikkert MO, Rockwood K. Frailty in elderly people. Lancet. 2013 Mar 2;381(9868):75262.

38. Zhang Y, Ge M, Zhao W, Hou L, Xia X, Liu X, Zuo Z, Zhao Y, Yue J, Dong B. Association Between Number of Teeth, Denture Use and Frailty: Findings from the West China Health and Aging Trend Study. J Nutr Health Aging. 2020;24(4):423-8.

39. Ramsay SE, Papachristou E, Watt RG, Tsakos G, Lennon LT, Papacosta AO, Moynihan P, Sayer AA, Whincup PH, Wannamethee SG. Influence of Poor Oral Health on Physical Frailty: A Population-Based Cohort Study of Older British Men. J Am Geriatr Soc. 2018 Mar;66(3):473-9.

40. Serra-Prat M, Sist X, Domenich R, Jurado L, Saiz A, Roces A, Palomera E, Tarradelles M, Papiol M. Effectiveness of an intervention to prevent frailty in pre-frail community-dwelling older people consulting in primary care: a randomised controlled trial. Age Ageing. 2017 May 1;46(3):401-7.

41. Ek K, Browall M, Eriksson M, Eriksson I. Healthcare providers' experiences of assessing and performing oral care in older adults. Int J Older People Nurs. 2018 Jun;13(2):e12189.

42. Croonquist CG, Dalum J, Skott P, Sjögren P, Wårdh I, Morén E. Effects of domiciliary professional oral care for care-dependent elderly in nursing homes-oral hygiene, gingival bleeding, root caries and nursing staff's oral health knowledge and attitudes. Clin Interv Aging. 2020 Aug; 15: 1305-15.

43. Sjögren P, Girestam CC, Skott P, Marsson N, Nova R, Zimmerman M, et al. Professional Domiciliary Oral Care for Elderly in Nursing Homes-A Randomized Controlled 
Pilot Trial. Health. 2016 Aug;8(11):1112.

44. Janssens B, Vanobbergen J, Petrovic M, Jacquet W, Schols JMGA, De Visschere L. The oral health condition and treatment needs assessment of nursing home residents in Flanders (Belgium). Community Dent Health. 2017 Sep;34(3):143-51.

45. Morin L, Johnell K, Laroche ML, Fastbom J, Wastesson JW. The epidemiology of polypharmacy in older adults: register-based prospective cohort study. Clin Epidemiol. 2018 Mar 12;10:289-98.

46. Alaee A, Azizi A, Valai N, Tarkashvand Dn. Dentist' Knowledge and Attitude Aabout Geriatrics' Dentisry. Teb Va Tazkieh. 2017;25(2):70-57.

47. Shiraishi A, Wakabayashi H, Yoshimura Y. Oral Management in Rehabilitation Medicine: Oral Frailty, Oral Sarcopenia, and Hospital-Associated Oral Problems. J Nutr Health Aging. 2020;24(10):1094-9.

48. Tynan A, Deeth L, McKenzie D. An integrated oral health program for rural residential aged care facilities: a mixed methods comparative study. BMC Health Services Research. 2018;18(1):1-12.

49. Janssens B, Vanobbergen J, Lambert M, Schols JMGA, De Visschere L. Effect of an oral healthcare programme on care staff knowledge and attitude regarding oral health: a non-randomised intervention trial. Clin Oral Investig. 2018 Jan;22(1):281-92.

50. Iwao Y, Shigeishi H, Takahashi S, Uchida S, Kawano $\mathrm{S}$, Sugiyama M. Improvement of physical and oral function in community $\square$ dwelling older people after a 3-month longterm care prevention program including physical exercise, oral health instruction, and nutritional guidance. Clin Exp Dent Res. 2019 Dec; 5(6):611-9.

\author{
Cite this paper as: Elaheh Aghaei, Mohammad \\ VahediArezoo Alaee. A review about oral health \\ and dental care among the aged population. $J$ \\ Res Dent Maxillofac Sci.2021;6 (3):30-38.
}

